\title{
A cautionary note on the use of Cook's distance
}

\author{
Myung Geun Kim ${ }^{1, a}$ \\ ${ }^{a}$ Department of Mathematics Education, Seowon University, Korea
}

\begin{abstract}
An influence measure known as Cook's distance has been used for judging the influence of each observation on the least squares estimate of the parameter vector. The distance does not reflect the distributional property of the change in the least squares estimator of the regression coefficients due to case deletions: the distribution has a covariance matrix of rank one and thus it has a support set determined by a line in the multidimensional Euclidean space. As a result, the use of Cook's distance may fail to correctly provide information about influential observations, and we study some reasons for the failure. Three illustrative examples will be provided, in which the use of Cook's distance fails to give the right information about influential observations or it provides the right information about the most influential observation. We will seek some reasons for the wrong or right provision of information.
\end{abstract}

Keywords: case deletion, Cook's distance, influence, regression

\section{Introduction}

In a regression context, there are many measures of the influence of observations on the least squares estimate of the parameter vector. Some of them can be found in Chatterjee and Hadi (1988) and Cook and Weisberg (1982). Since the change in the least squares estimate of regression coefficients due to case deletions is a vector quantity, it is usually normalized or scaled so that observations can be ordered in a certain way. In this vein Cook (1977) introduced an influence measure based on confidence ellipsoids.

The distribution of the change in the least squares estimator of the regression coefficients due to case deletions has a support set determined by a line in the multidimensional Euclidean space. Cook's distance does not reflect this kind of distributional property, and thus it can reduce or enlarge the influence of an observation on the least squares estimate. As a result, the use of Cook's distance is likely to underestimate the influence of an observation on the least squares estimate or overestimate it, which will be studied in Section 2. Hence the use of Cook's distance may lead to a wrong detection of influential observations. In Section 3 we consider three illustrative examples. For two examples, the use of Cook's distance fails to give the right information about influential observations, and for one example, it provides the right information about the most influential observation. We will seek some reasons for the wrong or right provision of information.

\footnotetext{
${ }^{1}$ Department of Mathematics Education, Seowon University, 377-3, Musimseo-ro, Seowon-gu, Cheongju-si, Chungcheongbuk-do 28674, Korea. E-mail: mgkim@seowon.ac.kr

Published 31 May 2017 / journal homepage: http://csam.or.kr

(c) 2017 The Korean Statistical Society, and Korean International Statistical Society. All rights reserved.
} 


\section{On Cook's distance}

A linear regression model of our interest can be expressed as

$$
y=X \beta+\varepsilon,
$$

where $y$ is an $n \times 1$ vector of values of the response variable, $X=\left(x_{1}, \ldots, x_{n}\right)^{T}$ is an $n \times p$ matrix of full column rank consisting of $n$ measurements on the $p$ fixed explanatory variables possibly including the intercept term, $\beta=\left(\beta_{0}, \beta_{1}, \ldots, \beta_{p-1}\right)^{T}$ is a $p \times 1$ vector of unknown regression coefficients, and $\varepsilon$ is an $n \times 1$ vector of independent random errors, each of which has zero mean and unknown variance $\sigma^{2}$. We write the least squares estimator of $\beta$ as $\hat{\beta}=\left(X^{T} X\right)^{-1} X^{T} y$ which is an unbiased estimator of $\beta$ and whose covariance matrix is given by $\operatorname{cov}(\hat{\beta})=\sigma^{2}\left(X^{T} X\right)^{-1}$. The $n \times n$ matrix $H=\left(h_{i j}\right)=X\left(X^{T} X\right)^{-1} X^{T}$ is the hat matrix, and $e=\left(e_{1}, \ldots, e_{n}\right)^{T}=(I-H) y$ is the residual vector. The mean of the residual vector $e$ is zero and its covariance matrix is $\operatorname{cov}(e)=\sigma^{2}(I-H)$. An unbiased estimator of $\sigma^{2}$ is given by $\hat{\sigma}^{2}=e^{T} e /(n-p)$. More details can be found in Seber (1977).

The least squares estimator of $\beta$ computed without observation $r$ is written as $\hat{\beta}_{(r)}$. Miller (1974) showed that

$$
\hat{\beta}-\hat{\beta}_{(r)}=\left(X^{T} X\right)^{-1} x_{r} \frac{e_{r}}{1-h_{r r}}, \quad r=1, \ldots, n
$$

The mean vector of $\hat{\beta}-\hat{\beta}_{(r)}$ is zero and its covariance matrix is

$$
\operatorname{cov}\left(\hat{\beta}-\hat{\beta}_{(r)}\right)=\frac{\sigma^{2}}{1-h_{r r}}\left(X^{T} X\right)^{-1} x_{r} x_{r}^{T}\left(X^{T} X\right)^{-1} .
$$

The rank of $\operatorname{cov}\left(\hat{\beta}-\hat{\beta}_{(r)}\right)$ is one for nonnull $x_{r}$. The only nonzero eigenvalue of $\operatorname{cov}\left(\hat{\beta}-\hat{\beta}_{(r)}\right)$ is $\sigma^{2} x_{r}^{T}\left(X^{T} X\right)^{-2} x_{r} /\left(1-h_{r r}\right)$ and its associated eigenvector is $\left(X^{T} X\right)^{-1} x_{r}$. When we denote by $V_{r}$ a onedimensional subspace generated by $\left(X^{T} X\right)^{-1} x_{r}$ of the $p$-dimensional Euclidean space, the subspace $V_{r}$ is just a line along which the eigenvector $\left(X^{T} X\right)^{-1} x_{r}$ lies, and each $\hat{\beta}-\hat{\beta}_{(r)}$ has a distribution with which a random variable takes on values in the set $V_{r}$ with probability one. More details about the distribution of $\hat{\beta}-\hat{\beta}_{(r)}$ can be found in $\operatorname{Kim}(2015)$.

In order to investigate the change in the value of $\hat{\beta}$ due to a deletion of observation $r$, Cook (1977) introduced an influence measure based on confidence ellipsoids as follows:

$$
D_{r}=\frac{1}{p \hat{\sigma}^{2}}\left(\hat{\beta}-\hat{\beta}_{(r)}\right)^{T}\left(X^{T} X\right)\left(\hat{\beta}-\hat{\beta}_{(r)}\right)
$$

Let $X^{T} X=G L G^{T}$ be the spectral decomposition of $X^{T} X$, where $L=\operatorname{diag}\left(l_{1}, \ldots, l_{p}\right)$ is a $p \times p$ diagonal matrix consisting of the eigenvalues of $X^{T} X, G=\left(g_{1}, \ldots, g_{p}\right)$ is a $p \times p$ orthogonal matrix, and $g_{i}$ is the eigenvector of $X^{T} X$ associated with the eigenvalue $l_{i}$. Then $X^{T} X=\sum_{i=1}^{p} l_{i} g_{i} g_{i}^{T}$. Hence $D_{r}$ can be expressed as

$$
\begin{aligned}
D_{r} & =\frac{1}{p \hat{\sigma}^{2}} \sum_{i=1}^{p} l_{i}\left[\left(\hat{\beta}-\hat{\beta}_{(r)}\right)^{T} g_{i}\right]^{2} \\
& =\frac{\left\|\hat{\beta}-\hat{\beta}_{(r)}\right\|^{2}}{p \hat{\sigma}^{2}} \sum_{i=1}^{p} l_{i} \cos ^{2} \theta_{r i},
\end{aligned}
$$


Table 1: The eigenvalues of $X^{T} X$

\begin{tabular}{ccccc}
\hline \hline$l_{1}$ & $l_{2}$ & $l_{3}$ & $l_{4}$ & $l_{5}$ \\
\hline 44676.2060 & 5965.4221 & 809.9521 & 105.4187 & 0.0012 \\
\hline \hline
\end{tabular}

where $\theta_{r i}$ is the angle between $\hat{\beta}-\hat{\beta}_{(r)}$ and $g_{i}$, and $\left\|\hat{\beta}-\hat{\beta}_{(r)}\right\|$ is the Euclidean norm of $\hat{\beta}-\hat{\beta}_{(r)}$.

The set $\left\{g_{1}, \ldots, g_{p}\right\}$ forms an orthonormal basis for the $p$-dimensional Euclidean space. The coordinate of $\hat{\beta}-\hat{\beta}_{(r)}$ with respect to the $i^{t h}$ eigenvector $g_{i}$ is $\left(\hat{\beta}-\hat{\beta}_{(r)}\right)^{T} g_{i}$. In the light of equation (2.1) the terms $l_{i}$ and $\left(\hat{\beta}-\hat{\beta}_{(r)}\right)^{T} g_{i}$ for each $r$ play a specific role in determining the magnitude of $D_{r}$. The adoption of $X^{T} X$ for scaling the Euclidean norm of $\hat{\beta}-\hat{\beta}_{(r)}$ is not reasonable as explained in what follows. In real data analyses, the line $V_{r}$ is not in general parallel to any of the eigenvectors $g_{1}, \ldots, g_{p}$. Also, even in the case where the line $V_{r}$ is almost parallel to one of the $g_{i}$ 's, it is nearly orthogonal to the other eigenvectors: for example, if the line $V_{r}$ is almost parallel to $g_{p}$, then the component of $D_{r}$ associated with the eigenvector $g_{p}, l_{p}\left[\left(\hat{\beta}-\hat{\beta}_{(r)}\right)^{T} g_{p}\right]^{2} / p \hat{\sigma}^{2}$ nearly makes a real contribution to the influence of observation $r$ on $\hat{\beta}$, while the component of $D_{r}$ associated with the remaining eigenvectors, $\sum_{i=1}^{p-1} l_{i}\left[\left(\hat{\beta}-\hat{\beta}_{(r)}\right)^{T} g_{i}\right]^{2} / p \hat{\sigma}^{2}$ is likely to distort the influence of observation $r$ on $\hat{\beta}$. Most or all of the terms $\left(\hat{\beta}-\hat{\beta}_{(r)}\right)^{T} g_{i}$ for each observation are computed in the outside of the set $V_{r}$ over which $\hat{\beta}-\hat{\beta}_{(r)}$ is distributed. As a result, the terms $\left(\hat{\beta}-\hat{\beta}_{(r)}\right)^{T} g_{i}$ play a role of having the value of $D_{r}$ reduced or enlarged depending on the values of $l_{i}$ as compared with the real influence of observation $r$, which results in distorting the influence of observation $r$. Hence the use of $D_{r}$ is likely to underestimate the influence of observation $r$ on $\hat{\beta}$ or overestimate it, and the information about influential observations that the Cook's distance provides may not be reliable.

\section{Three illustrative examples}

We will apply the expressions of $D_{r}$ in equation (2.1) to three data sets: the Hald data set (Draper and Smith, 1981) and the rat data set (Cook and Weisberg, 1982) which were analyzed also by Cook (1977), and the body fat data set (Neter et al., 1996, p. 261). Using the probabilistic behavior of $\hat{\beta}-\hat{\beta}_{(r)}$ through the spectral decomposition of its covariance matrix $\operatorname{cov}\left(\hat{\beta}-\hat{\beta}_{(r)}\right)$, Kim (2015) introduced an influence measure $M_{r}=x_{r}^{T}\left(X^{T} X\right)^{-2} x_{r} /\left(1-h_{r r}\right)$ to investigate the influence of deleting an observation on the least squares estimate $\hat{\beta}$, and the problem of deleting multiple cases was considered by Kim (2016). For these three data sets, the result based on the $D_{r}$ values will be compared with that based on the $M_{r}$ values.

\subsection{Hald data}

The regression model with the intercept term $\beta_{0}$ is fitted to the Hald data set which consists of 13 observations on a single dependent variable and four independent variables. The estimated regression coefficients are $\hat{\beta}_{0}=62.41, \hat{\beta}_{1}=1.55, \hat{\beta}_{2}=0.51, \hat{\beta}_{3}=0.10$, and $\hat{\beta}_{4}=-0.14$.

For the values of $D_{r}$, observation 8 has the largest value $D_{8}=0.394$ and observation 3 has the second largest value $D_{3}=0.301$. Based on the $D_{r}$ values, observation 8 is thus identified as the most influential observation. However, the influence measure $M_{r}$ shows that observation 3 has the largest influence on the least squares estimate of $\beta\left(M_{3}=879.74\right)$ but observation 8 is not significantly influential $\left(M_{8}=37.16\right)$. In order to seek some reasons for which the two results are contradictory to each other, we will investigate sources of the $D_{r}$ values for observations 3 and 8 . The eigenvalues of $X^{T} X$ and their associated eigenvectors are included in Tables 1 and 2, respectively.

(a) Each row in Table 3 shows a normalized vector of each $\hat{\beta}-\hat{\beta}_{(r)}$. The values of $\cos \theta_{r i}$ shown in 
Table 2: The eigenvectors of $X^{T} X$

\begin{tabular}{crrrr}
\hline \hline$g_{1}$ & $g_{2}$ & $g_{3}$ & \multicolumn{1}{c}{$g_{4}$} & $g_{5}$ \\
\hline-0.01699 & 0.00372 & 0.00004 & -0.01104 & 0.99979 \\
-0.12789 & -0.04278 & -0.64590 & -0.75134 & -0.01028 \\
-0.83968 & -0.50922 & -0.01812 & 0.18763 & -0.01030 \\
-0.19842 & 0.07211 & 0.75572 & -0.61985 & -0.01052 \\
-0.48881 & 0.85653 & -0.10665 & 0.12626 & -0.01010 \\
\hline \hline
\end{tabular}

Table 3: Normalized vectors of $\hat{\beta}-\hat{\beta}_{(r)}$

\begin{tabular}{|c|c|c|c|c|c|}
\hline Number & & & & & \\
\hline 1 & -0.99982 & 0.00772 & 0.01084 & 0.00706 & 0.01158 \\
\hline 2 & 0.99976 & -0.01306 & -0.01003 & -0.01155 & -0.00904 \\
\hline 3 & -0.99979 & 0.01002 & 0.01021 & 0.01065 & 0.01013 \\
\hline 4 & -0.99981 & 0.00799 & 0.01083 & 0.01008 & 0.00964 \\
\hline 5 & -0.99984 & 0.00158 & 0.01304 & 0.00142 & 0.01216 \\
\hline 6 & 0.99982 & -0.00691 & -0.01005 & -0.01004 & -0.01052 \\
\hline 7 & 0.99981 & -0.00095 & -0.01376 & -0.01099 & -0.00820 \\
\hline 8 & 0.99969 & -0.01306 & -0.00889 & -0.01656 & -0.00986 \\
\hline 9 & 0.99979 & -0.01122 & -0.01009 & -0.00975 & -0.01024 \\
\hline 10 & -0.99952 & 0.02344 & 0.00807 & 0.01604 & 0.00885 \\
\hline 11 & -0.99972 & 0.01230 & 0.00941 & 0.01523 & 0.00958 \\
\hline 12 & -0.99979 & 0.01058 & 0.01074 & 0.01015 & 0.00981 \\
\hline 13 & 0.99981 & -0.00893 & -0.01118 & -0.00874 & -0.01007 \\
\hline
\end{tabular}

Table 4: The values of $\cos \theta_{r i}$

\begin{tabular}{|c|c|c|c|c|c|}
\hline \multirow{2}{*}{$r$} & \multicolumn{5}{|c|}{$i$} \\
\hline & 1 & 2 & 3 & 4 & 5 \\
\hline 1 & -0.00016 & 0.00085 & -0.00112 & 0.00436 & -0.99999 \\
\hline 2 & -0.00019 & 0.00081 & 0.00090 & 0.00291 & 1.00000 \\
\hline 3 & 0.00007 & 0.00010 & 0.00027 & 0.00010 & -1.00000 \\
\hline 4 & 0.00017 & -0.00060 & 0.00119 & 0.00204 & -1.00000 \\
\hline 5 & -0.00038 & 0.00009 & -0.00152 & 0.01296 & -0.99991 \\
\hline 6 & -0.00053 & -0.00059 & -0.00178 & -0.00284 & 0.99999 \\
\hline 7 & 0.00087 & 0.00295 & -0.00653 & -0.00713 & 0.99995 \\
\hline 8 & 0.00025 & -0.00084 & -0.00282 & 0.00612 & 0.99998 \\
\hline 9 & -0.00014 & -0.00013 & 0.00120 & 0.00024 & 1.00000 \\
\hline 10 & -0.00030 & -0.00009 & -0.00415 & -0.01389 & -0.99989 \\
\hline 11 & -0.00019 & 0.00027 & 0.00234 & -0.00467 & -0.99999 \\
\hline 12 & -0.00019 & -0.00051 & -0.00045 & 0.00006 & -1.00000 \\
\hline 13 & 0.00020 & 0.00054 & 0.00048 & -0.00228 & 1.00000 \\
\hline
\end{tabular}

Table 5: The ratios $\cos ^{2} \theta_{8 i} / \cos ^{2} \theta_{3 i}$

\begin{tabular}{cccc}
\hline \hline 1 & & & \\
& & & \\
& 2 & 3 & 3 \\
\hline 12.8 & 73.6 & 109.5 & 3,955 \\
\hline \hline
\end{tabular}

Table 4 can be considered as a measure of closeness of $\hat{\beta}-\hat{\beta}_{(r)}$ to the $i^{\text {th }}$ eigenvector $g_{i}$ of $X^{T} X$. As $\hat{\beta}-\hat{\beta}_{(r)}$ gets close to $g_{i}$, the value of $\cos \theta_{r i}$ approaches to one. We note from Tables 2 and 3 that both vectors $\hat{\beta}-\hat{\beta}_{(3)}$ and $\hat{\beta}-\hat{\beta}_{(8)}$ are almost parallel to the last eigenvector $g_{5}$ of $X^{T} X$, which can be confirmed by the $\cos \theta_{r i}$ values shown in the last column of Table 4 . The second to fifth columns of Table 4 show that both vectors $\hat{\beta}-\hat{\beta}_{(3)}$ and $\hat{\beta}-\hat{\beta}_{(8)}$ are almost orthogonal to each of the eigenvectors $g_{1}, \ldots, g_{4}$ of $X^{T} X$.

(b) For observations 3 and 8 , the ratios $\cos ^{2} \theta_{8 i} / \cos ^{2} \theta_{3 i}$ listed in Table 5 are much larger than one 
Table 6: Two components of Cook's distance for observations 3 and 8

\begin{tabular}{cccc}
\hline \hline$r$ & $D_{r}$ & $\sum_{i=1}^{4} l_{i}\left[\left(\hat{\beta}-\hat{\beta}_{(r)}\right)^{T} g_{i}\right]^{2} / p \hat{\sigma}^{2}$ & $l_{5}\left[\left(\hat{\beta}-\hat{\beta}_{(r)}\right)^{T} g_{5}\right]^{2} / p \hat{\sigma}^{2}$ \\
\hline 3 & 0.301 & 0.065 & 0.236 \\
8 & 0.394 & 0.368 & 0.026 \\
\hline \hline
\end{tabular}

Table 7: The change in $\hat{\beta}_{k}$ due to deletion of each of observations 3 and 8

\begin{tabular}{cccccc}
\hline \hline$r$ & $\hat{\beta}_{0}-\hat{\beta}_{0(r)}$ & $\hat{\beta}_{1}-\hat{\beta}_{1(r)}$ & $\hat{\beta}_{2}-\hat{\beta}_{2(r)}$ & $\hat{\beta}_{3}-\hat{\beta}_{3(r)}$ & $\hat{\beta}_{4}-\hat{\beta}_{4(r)}$ \\
\hline 3 & -76.18 & 0.76 & 0.78 & 0.81 & 0.77 \\
8 & 25.16 & -0.33 & -0.22 & -0.42 & -0.25 \\
\hline \hline
\end{tabular}

for all $i=1,2,3,4$, and hence they show that observation 8 is located closer to all of four axes $g_{1}, \ldots, g_{4}$ than observation 3 .

(c) Since the line $V_{3}$ over which $\hat{\beta}-\hat{\beta}_{(3)}$ is distributed is almost parallel to the eigenvector $g_{5}$ of $X^{T} X$, the component of $D_{3}$ associated with the eigenvector $g_{5}$ which is $l_{5}\left[\left(\hat{\beta}-\hat{\beta}_{(3)}\right)^{T} g_{5}\right]^{2} / p \hat{\sigma}^{2}$ nearly makes a real contribution to the influence of observation 3 on $\hat{\beta}$. These components of $D_{r}(r=3,8)$ are included in Table 6. Also, the proportion of $l_{5}\left[\left(\hat{\beta}-\hat{\beta}_{(r)}\right)^{T} g_{5}\right]^{2} / p \hat{\sigma}^{2}$ to $D_{r}$ is about $79 \%$ for observation 3 and about $7 \%$ for observation 8 . On the other hand, the line $V_{3}$ is almost orthogonal to all of the eigenvectors $g_{1}, \ldots, g_{4}$ of $X^{T} X$, and therefore the component of $D_{3}$ associated with the eigenvectors $g_{1}, \ldots, g_{4}$ which is $\sum_{i=1}^{4} l_{i}\left[\left(\hat{\beta}-\hat{\beta}_{(3)}\right)^{T} g_{i}\right]^{2} / p \hat{\sigma}^{2}$ is likely to distort the influence of observation 3 on $\hat{\beta}$. Observation 8 can be interpreted similarly to observation 3 . The difference of $\sum_{i=1}^{4} l_{i}\left[\left(\hat{\beta}-\hat{\beta}_{(r)}\right)^{T} g_{i}\right]^{2} / p \hat{\sigma}^{2}$ between observations 3 and 8 is approximately -0.303 , while the difference of $l_{5}\left[\left(\hat{\beta}-\hat{\beta}_{(r)}\right)^{T} g_{5}\right]^{2} / p \hat{\sigma}^{2}$ between observations 3 and 8 is approximately 0.210 . The extent that the distance $D_{8}$ distorts the influence of observation 8 on $\hat{\beta}$ is far more severe than that of $D_{3}$. Thus the component $\sum_{i=1}^{4} l_{i}\left[\left(\hat{\beta}-\hat{\beta}_{(r)}\right)^{T} g_{i}\right]^{2} / p \hat{\sigma}^{2}$ plays a role of making the value of $D_{8}$ large, while it plays a role of making the value of $D_{3}$ relatively small. Hence the distance $D_{8}$ enlarges the influence of observation 8 on $\hat{\beta}$, while the distance $D_{3}$ reduces the influence of observation 3 .

This is a reason for which the use of the $D_{r}$ values identifies observation 8 that is not significantly influential as the most influential one and it cannot detect observation 3 as the most influential one.

Even though the $D_{r}$ value was introduced as an overall measure of the combined influence of observation $r$ on all of the estimated regression coefficients, it would be desirable if the use of the $D_{r}$ values reveals influential observations for each regression coefficient, but the use of the $D_{r}$ values does not. The use of the $D_{r}$ values asserts that deletion of observation 8 has the largest change in $\hat{\beta}$. However, deletion of observation 8 does not bring about a significant change in either estimated regression coefficient, while deletion of observation 3 has the largest change in all of the estimated regression coefficients, as can be seen in what follows. Numerical computations of the values $\hat{\beta}_{k}-$ $\hat{\beta}_{k(r)}, k=0,1, \ldots, 4 ; r=1, \ldots, 13$ show that deletion of observation 3 has the largest change in $\hat{\beta}_{k}$ for all $k=0,1, \ldots, 4$. Table 7 shows the change in $\hat{\beta}_{k}$ due to deletion of each of observations 3 and 8 . After removal of observation 8 from the sample, numerical computations based on the remaining sample of size 12 show that deletion of observation 3 still has the largest change in $\hat{\beta}_{k}$ for all $k=0,1, \ldots, 4$ as listed in Table 8. After removal of observation 3 from the sample, numerical computations based on the remaining sample of size 12 show that deletion of observation 4 has the largest change -75.77 in $\hat{\beta}_{0}$, deletion of observation 11 has the largest change 0.77 in $\hat{\beta}_{1}$, deletion of observation 4 has the largest change 0.79 in $\hat{\beta}_{2}$, deletion of observation 11 has the largest change 0.90 in $\hat{\beta}_{3}$, and deletion of 
Table 8: The change in $\hat{\beta}_{k}$ due to deletion of observations 3 after removal of observation 8 from the sample

\begin{tabular}{ccccc}
\hline \hline$\hat{\beta}_{0}-\hat{\beta}_{0(r)}$ & $\hat{\beta}_{1}-\hat{\beta}_{1(r)}$ & $\hat{\beta}_{2}-\hat{\beta}_{2(r)}$ & $\hat{\beta}_{3}-\hat{\beta}_{3(r)}$ & $\hat{\beta}_{4}-\hat{\beta}_{4(r)}$ \\
\hline-49.17 & 0.50 & 0.50 & 0.54 & 0.50 \\
\hline \hline
\end{tabular}

observation 4 has the largest change 0.75 in $\hat{\beta}_{4}$. We note that the $M_{r}$ values provide useful information about influential observations for each regression coefficient.

\subsection{Body fat data}

We fit the regression model with the intercept term $\beta_{0}$ to the the body fat data set which has 20 measurements on a single dependent variable and three independent variables. The least squares estimates of the regression coefficients are $\hat{\beta}_{0}=117.08, \hat{\beta}_{1}=4.33, \hat{\beta}_{2}=-2.86$, and $\hat{\beta}_{3}=-2.19$.

Observation 3 has the largest value $D_{3}=0.299$ and observation 1 has the second largest distance $D_{1}=0.279$. The $D_{r}$ values assert that observation 3 is the most influential observation. However, for the $M_{r}$ values, observation 1 has the largest value $M_{1}=401.19$ and observation 3 has $M_{3}=$ 150.22 , not the second largest value. We have contradictory results also for the body fat data. We will seek some reasons for this contradictory results by investigating sources of the $D_{r}$ values for observations 1 and 3. Detailed computations will not be included here. The four eigenvalues of $X^{T} X$ are $81290.24,294.25,119.82,0.00062$. The eigenvector corresponding to the last eigenvalue is $(0.99909,0.03012,-0.02583,-0.01592)$. Euclidean norm $\left\|\hat{\beta}-\hat{\beta}_{(r)}\right\|$ is 72.92 for observation 1 and 37.47 for observation 3 .

(a) An investigation of the closeness between a normalized vector of each $\hat{\beta}-\hat{\beta}_{(r)}$ and each eigenvector of $X^{T} X$ shows that $\cos \theta_{r 4}$ is -0.9999988 for observation 1 and 0.9999891 for observation 3, which implies that both vectors $\hat{\beta}-\hat{\beta}_{(1)}$ and $\hat{\beta}-\hat{\beta}_{(3)}$ are almost parallel to the last eigenvector $g_{4}$ of $X^{T} X$. Also, both vectors $\hat{\beta}-\hat{\beta}_{(1)}$ and $\hat{\beta}-\hat{\beta}_{(3)}$ are almost orthogonal to each of the remaining eigenvectors of $X^{T} X$.

(b) For observations 1 and 3, the ratio $\cos ^{2} \theta_{3 i} / \cos ^{2} \theta_{1 i}$ is $5.09,5.18,15.26$ for $i=1,2,3$, respectively. Hence observation 3 is located closer to all of three axes $g_{1}, g_{2}, g_{3}$ than observation 1 .

(c) In the light of the results in (a), among the components of $D_{r}(r=1,3)$ given in the first expression of equation (2.1), only the component

$$
\frac{l_{4}\left[\left(\hat{\beta}-\hat{\beta}_{(r)}\right)^{T} g_{4}\right]^{2}}{p \hat{\sigma}^{2}}
$$

nearly makes a real contribution to the influence of observation $r$ on $\hat{\beta}$, and its value is 0.133 for observation 1 and 0.035 for observation 3. Also, the proportion of $l_{4}\left[\left(\hat{\beta}-\hat{\beta}_{(r)}\right)^{T} g_{4}\right]^{2} / p \hat{\sigma}^{2}$ to $D_{r}$ is about $48 \%$ for observation 1 and about $12 \%$ for observation 3 . On the other hand, since the line $V_{r}(r=1,3)$ is almost orthogonal to all of the remaining eigenvectors $g_{1}, g_{2}, g_{3}$ of $X^{T} X$, the component of $D_{r}$ associated with the eigenvectors $g_{1}, g_{2}, g_{3}$ which is

$$
\frac{1}{p \hat{\sigma}^{2}} \sum_{i=1}^{3} l_{i}\left[\left(\hat{\beta}-\hat{\beta}_{(r)}\right)^{T} g_{i}\right]^{2}
$$


is likely to distort the influence of observation $r$ on $\hat{\beta}$. The component $\sum_{i=1}^{3} l_{i}\left[\left(\hat{\beta}-\hat{\beta}_{(r)}\right)^{T} g_{i}\right]^{2}$ is 0.146 for observation 1 and 0.264 for observation 3 . The extent that the distance $D_{3}$ distorts the influence of observation 3 on $\hat{\beta}$ is more severe than that of $D_{1}$. The component $\sum_{i=1}^{3} l_{i}\left[\left(\hat{\beta}-\hat{\beta}_{(r)}\right)^{T} g_{i}\right]^{2} / p \hat{\sigma}^{2}$ plays a role of making the value of $D_{3}$ large, while it plays a role of making the value of $D_{1}$ relatively small. Hence the distance $D_{3}$ enlarges the influence of observation 3 on $\hat{\beta}$, while the distance $D_{1}$ reduces the influence of observation 1 .

This is a reason why observation 3 has the largest $D_{r}$ value, $D_{3}=0.299$, though it is not identified as a significantly influential observation by the $M_{r}$ values.

Furthermore, the $D_{r}$ values do not provide useful information about influential observations for each regression coefficient but the $M_{r}$ values do as can be seen in what follows. Numerical computations of the values $\hat{\beta}_{k}-\hat{\beta}_{k(r)}, k=0,1,2,3 ; r=1, \ldots, 20$ show that deletion of observation 1 has the largest change in $\hat{\beta}_{k}$ for all $k=0,1,2,3: \hat{\beta}_{0}-\hat{\beta}_{0(r)}$ is -72.86 for observation 1 and 37.44 for observation $3, \hat{\beta}_{1}-\hat{\beta}_{1(r)}$ is -2.12 for observation 1 and 1.02 for observation $3, \hat{\beta}_{2}-\hat{\beta}_{2(r)}$ is 1.88 for observation 1 and -0.87 for observation $3, \hat{\beta}_{3}-\hat{\beta}_{3(r)}$ is 1.08 for observation 1 and -0.69 for observation 3. Observation 3 is identified as the most influential one by the $D_{r}$ values but it does not have a significant influence on any estimate $\hat{\beta}_{k}(k=0,1,2,3)$.

\subsection{Rat data}

The regression model with the intercept term $\beta_{0}$ is fitted to the rat data set which consists of 19 measurements on a single dependent variable and three independent variables. The least squares estimates of the regression coefficients are $\hat{\beta}_{0}=0.27, \hat{\beta}_{1}=-0.02, \hat{\beta}_{2}=0.01$, and $\hat{\beta}_{3}=4.18$.

For the $D_{r}$ values, observation 3 has the largest value $D_{3}=0.930$. For the $M_{r}$ values, observation 3 has the largest value $M_{3}=1864.3$. Both influence measures lead to the same conclusion that observation 3 is the most influential one. We will briefly seek some reasons for the same conclusion. Detailed computations will not be included here. The four eigenvalues of $X^{T} X$ are 565097.6, 20.5, 0.16, 0.003. The eigenvector $g_{4}$ corresponding to the last eigenvalue is $(0.0213,-0.0052,0.0005,0.9998)$. For observation 3, we have Euclidean norm $\left\|\hat{\beta}-\hat{\beta}_{(3)}\right\|=2.684$.

The cosine of the angle between $\hat{\beta}-\hat{\beta}_{(3)}$ and $g_{4}$ is 0.9993 , which implies that $\hat{\beta}-\hat{\beta}_{(3)}$ is almost parallel to the last eigenvector $g_{4}$ of $X^{T} X$ and that it is almost orthogonal to each of the remaining eigenvectors of $X^{T} X$. Hence, among the components of $D_{3}$, only the component $l_{4}\left[\left(\hat{\beta}-\hat{\beta}_{(3)}\right)^{T} g_{4}\right]^{2} / p \hat{\sigma}^{2}$ nearly makes a real contribution to the influence of observation 3 on $\hat{\beta}$, and its value is 0.781 . The component $\sum_{i=1}^{3} l_{i}\left[\left(\hat{\beta}-\hat{\beta}_{(3)}\right)^{T} g_{i}\right]^{2}$ is 0.149 . Also, the proportion of $l_{4}\left[\left(\hat{\beta}-\hat{\beta}_{(3)}\right)^{T} g_{4}\right]^{2} / p \hat{\sigma}^{2}$ to $D_{3}$ is about $84 \%$ and it is very high. Therefore the extent that the distance $D_{3}$ reflects the real influence of observation 3 on $\hat{\beta}$ is very high so that the value $D_{3}$ can yield the same result as the value $M_{3}$.

\section{Concluding remarks}

The distance $D_{r}$ is defined by scaling $\hat{\beta}-\hat{\beta}_{(r)}$ using the matrix $X^{T} X$. Almost all of the eigenvectors $X^{T} X$ are not in general parallel to the line $V_{r}$ over which $\hat{\beta}-\hat{\beta}_{(r)}$ is distributed. The distance $D_{r}$ inevitably includes the component associated with the axes other than the axis determined by the line $V_{r}$. This component of $D_{r}$ can be a source of distorting the influence of observation $r$ on $\hat{\beta}$. Hence the information about influential observations that the Cook's distance provides may not be reliable. The first two examples analyzed in the previous section show defects of the distance $D_{r}$ as an influence measure, while the three examples show that the $M_{r}$ values can be a useful influence measure. 


\section{References}

Chatterjee S and Hadi AS (1988). Sensitivity Analysis in Linear Regression, Wiley, New York.

Cook RD (1977). Detection of influential observation in linear regression, Technometrics, 19, 15-18.

Cook RD and Weisberg S (1982). Residuals and Influence in Regression, Chapman and Hall, New York.

Draper NR and Smith H (1981). Applied Regression Analysis (2nd ed), Wiley, New York.

Kim MG (2015). Influence measure based on probabilistic behavior of regression estimators, Computational Statistics, 30, 97-105.

Kim MG (2016). Deletion diagnostics in fitting a given regression model to a new observation, Communications for Statistical Applications and Methods, 23, 231-239.

Miller RG (1974). An unbalanced jackknife, Annals of Statistics, 2, 880-891.

Neter J, Kutner MH, Nachtsheim CJ, and Wasserman W (1996). Applied Linear Regression Models (3rd ed), McGraw-Hill, Irwin.

Seber GAF (1977). Linear Regression Analysis, Wiley, New York. 